\title{
CARACTERIZACIÓN CLINICOEPIDEMIOLÓGICA DE PACIENTES CON ENFERMEDAD RENAL CRÓNICA, RIOBAMBA, 2021
}

\author{
CLINICALEPIDEMIOLOGICAL CHARACTERIZATION OF PATIENTS WITH CHRONIC \\ KIDNEY DISEASE, RIOBAMBA 2021
}

\author{
María Robalino Rivadeneira ${ }^{(1)}$; Geritza Urdaneta Carruyo ${ }^{(2)}$; Raquel Robalino Gualoto \\ ${ }^{(1)}$; Marlon Cobos Castillo ${ }^{(1)}$; Karla Andrade Zuña ${ }^{(1)}$; Cecilia Chanaguano Tixelema ${ }^{(1)}$. \\ (1) Hospital Provincial General Docente, Riobamba - Ecuador. \\ (2) Universidad del Zulia-Facultad de Medicina, Zulia - Venezuela.
}

Email:cecilia1994consuelo@gmail.com

https://doi.org/10.33789/talentos.8.2.154

\begin{abstract}
Resumen: La enfermedad renal crónica es uno de los grandes retos para los profesionales de la salud; constituye un problema de salud que genera discapacidad funcional, disminución de calidad de vida y elevados costos a los sistemas de salud. El objetivo de la presente investigación fue caracterizar clinicoepidemiológicamente a los pacientes con diagnóstico de enfermedad renal crónica atendidos en el Hospital Provincial General Docente de Riobamba. Para esto se desarrolló una investigación básica, descriptiva y de corte transversal. El universo estuvo constituido por 96 pacientes con diagnóstico de enfermedad renal crónica y la muestra quedó conformada por un total de 78 pacientes. Se aplicó un cuestionario que permitió identificar las características clínicas y epidemiológicas de los pacientes incluidos en el estudio. Los principales resultados incluyen un promedio de edad de 54,68 años. Predominaron las pacientes femeninas (60,26\%). El 98,72\% de ellos presento al menos una comorbilidad asociada, donde la hipertensión arterial $(92,21 \%)$ y la diabetes mellitus $(35,06 \%)$ fueron las más representadas. El 42,31\% de los pacientes presentó trastornos nutricionales por exceso. Se concluye que el patrón clínico epidemiológico de los pacientes con enfermedad renal crónica coincide con los elementos reportados en la literatura. Sin embargo, existen elementos como la presencia de la enfermedad en menores de 40 años y el elevado porcentaje de trastornos nutricionales por
\end{abstract}

Recibido: 02 de mayo de 2021

Online: 30 de noviembre de 2021

Publicado como artículo científico en la Revista de Investigación Talentos 8 (2), 56-67
Aceptado: 25 de noviembre de 2021
Publicación: 01 de julio de 2021 
exceso, que constituyen señales de alarma para los directivos de salud y para los profesionales en torno a la promoción de salud y prevención de enfermedades.

Palabras Claves: Caracterización epidemiológica, Calidad de vida, Enfermedad renal crónica, Insuficiencia renal crónica, Manifestaciones clínicas

\begin{abstract}
Chronic kidney disease is one of the great challenges for health professionals; it constitutes a health problem that generates functional disability, decreased quality of life and high costs to health systems. The objective of the present research was to characterize clinicoepidemiologically patients with a diagnosis of chronic kidney disease treated at the Riobamba Provincial General Teaching Hospital. For this, a basic, descriptive and crosssectional research was developed. The universe consisted of 96 patients with a diagnosis of chronic kidney disease and the sample was made up of a total of 78 patients. A questionnaire was applied to identify the clinical and epidemiological characteristics of the patients included in the study. The main results include an average age of 54.68 years. Female patients predominated (60.26\%). 98.72\% of them presented at least one associated comorbidity, where arterial hypertension (92.21\%) and diabetes mellitus (35.06\%) were the most represented. $42.31 \%$ of the patients presented nutritional disorders due to excess. It is concluded that the epidemiological clinical pattern of patients with chronic kidney disease coincides with the elements reported in the literature. However, there are elements such as the presence of the disease in people under 40 years of age and the high percentage of nutritional disorders due to excess, which constitute alarm signals for health managers and for professionals around health promotion and prevention of diseases.
\end{abstract}

Keywords: Epidemiological characterization, Quality of life, Chronic kidney disease, Chronic kidney failure, Clinical manifestations

\title{
I. INTRODUCCIÓN
}

El control de las enfermedades crónicas no transmisibles (ECNT) es descrito como uno de los retos más importantes para los profesionales de la salud a nivel mundial. Se describe que este tipo de enfermedades constituyen la segunda causa de mortalidad a nivel general y las distintas enfermedades que la componen repercuten negativamente en la capacidad funcional y la percepción de calidad de vida de los pacientes (Serra Valdés,
Serra Ruíz, \& Viera García, 2018).

Las ECNT afectan cualquier órgano o sistema de órganos del cuerpo humano; las más conocidas son la hipertensión arterial, la diabetes mellitus, el asma bronquial, la artritis reumatoide y los trastornos renales entre otras (Mora Marcial, Verdecia Tamallo, Rodríguez Vergara, del Pino, \& Guerra Cabrera, 2017). Dentro de este grupo destaca, por el grado de complejidad de la atención de salud que 
demanda, la enfermedad renal crónica (ERC). La ERC se caracteriza por la disminución de la función renal, puede ser secundaria a otras enfermedades crónicas como la hipertensión arterial, la diabetes mellitus, la obesidad y otras alteraciones renales primarias (Gansevoort, et al, 2013). A su vez, también se comporta la ERC como un factor de riesgo de aparición de otras enfermedades crónicas como la enfermedad cardiovascular, la propia hipertensión arterial y otros trastornos cardiovasculares entre otras (Otzen, Fuentes, Wetzel, Henríquez, Antúnez, \& Melnik, 2020).

Se describe como principal componente fisiopatológico de la ERC la disminución de la tasa de filtración glomerular (TFG) que es independiente de la edad, sexo y de otros factores deriesgo.Ladisminución delafunción renal es el principal elemento diagnóstico de la enfermedad y un factor considerado como predictor de hospitalización, de disfunción cognitiva y de afectación considerable de la calidad de vida (Gutiérrez Rufín, \& Polanco López, 2018).

La ERC se define como la pérdida progresiva, permanente e irreversible de la TFG a lo largo de un tiempo variable, a veces incluso de años, expresada por una reducción del aclaramiento de creatinina estimado inferior a los $60 \mathrm{ml} / \mathrm{min} / 1,73 \mathrm{~m}^{2} \mathrm{SC}$. A medida que progresa la enfermedad va disminuyendo cada vez más la TFG hasta llegar a estadios avanzados donde el paciente necesita de terapia sustitutiva renal, ya sea por diálisis o trasplante renal (Díaz Armas, Gómez Leyva, Robalino Valdivieso, \& Lucero Proaño, 2018).
Se reporta que la ERC puede afectar a personas de cualquier edad y sexo, existen patrones de presentación que pudieran considerarse como característicos de la enfermedad. Se describe que en pacientes masculinos la principal causa de ERC se asocia a enfermedades metabólicas como la gota o el síndrome metabólico y por eso existen distintos perfiles epidemiológicos y clínicos posibles. En las féminas con ERC sucede similar y se plantean como posibles causas la hipertensión arterial, la artritis reumatoide, la diabetes mellitus y el lupus eritematoso sistémico entre otras (Torres Rondón, Bandera Ramos, Ge Martínez, \& Amaro Guerra, 2017). Sin embargo, a pesar de ser variable el componente epidemiológico y clínico de la enfermedad, si existe coincidencia en señalar la gran afectación que genera la enfermedad a la capacidad funcional y calidad de vida de los pacientes, así como los gastos económicos de atención médica y afectación social que se genera (Poll Pineda, Rueda Macías, Poll Rueda, Mancebo Villalón, \& Arias Moncada, 2017).

Es por eso que, teniendo en cuenta la afectación que genera la ERC en la capacidad funcional y la calidad de vida de los pacientes, los gastos de salud que genera y la variedad de características clínicas y epidemiológicas que se presentan durante su establecimiento y evolución; se decide realizar esta investigación con el objetivo de caracterizar clinicoepidemiológicamente a los pacientes con diagnóstico de ERC atendidos en el Hospital Provincial General Docente de Riobamba (HPGDR) durante el primer semestre del año 2021, lo que permitirá mejorar la orientación diagnostica 
esta enfermedad.

\section{MATERIALES Y MÉTODOS}

Serealizó una investigación básica, descriptiva y de corte transversal que incluyó un enfoque mixto al utilizar elementos cualitativos $\mathrm{y}$ cuantitativos. El universo estuvo constituido por 96 pacientes con diagnóstico de ERC según los criterios la Organización Mundial de Salud. Para calcular el tamaño de la muestra se utilizó la fórmula matemática para poblaciones conocidas. Después de realizar el cálculo matemático se identificó que la muestra estaría conformada por 78 pacientes. Se utilizó el método aleatorio simple para conformar la muestra de la investigación, lo que garantizó que todos los pacientes con diagnóstico de ERC tuvieran la misma posibilidad de participar en la investigación. Cada uno de los pacientes que finalmente fueron incluidos en el estudio expresó su deseo de participar en el estudio mediante la firma del consentimiento informado y tenían confirmado el diagnóstico de ERC; los cuales fueron los únicos criterios de inclusión definidos para la investigación.

Se definieron dos variables de investigación; la primera de ellas fue la variable características epidemiológicas que incluyó subvariables como la edad, sexo, procedencia, autoidentificación étnica, presencia de comorbilidades y tipo de comorbilidades. La segunda variable fue denominada como características clínicas e incluyo subvariables como tiempo de evolución de la enfermedad, tipo de manifestaciones clínicas y valores de función renal determinados por las cifras de urea, creatinina y ácido úrico del último control realizado a los pacientes.

Se utilizaron como técnicas de investigación la revisión documental y la entrevista. La revisión documental consistió en el análisis minucioso de la historia clínica de cada paciente incluido en el estudio para obtener información relacionada con los valores de función renal y aclarar dudas o términos de difícil manejo por parte de los pacientes. Por su parte la entrevista facilitó obtener información necesaria para dar cumplimiento a las variables y objetivos del estudio.

Como cuestionario de investigación se utilizó una encuesta diseñada específicamente para el estudio. Este fue sometido, previa a su aplicación, al análisis de un comité de expertos conformados por 5 especialistas en metodología de la investigación, medicina interna, administración de salud y nefrología. Después de una segunda ronda de revisión los expertos emitieron un criterio favorable para la aplicación del instrumento. La encuesta tuvo un total de 17 preguntas divididas en dos sesiones. La primera sesión incluyó 8 preguntas orientadas a determinar los elementos epidemiológicos definidos. La segunda sesión constó con 9 preguntas y se orientaron a la identificación de las características clínicas. Se utilizó una hoja de recolección de información donde se incorporaron todos los datos obtenidos.

El procesamiento de la información se realizó de forma automatizada con la ayuda del programa estadístico SPSS en su versión 26 para Windows. Se definieron medidas de tendencia central y de dispersión para el 
procesamiento de las variables cuantitativas y frecuencias absolutas y porcentajes en el caso de las variables cualitativas. El nivel de confianza fue definido en el $95 \%$ y el margen de error en el $5 \%$. Todos los resultados fueron expresados en forma de tablas estadísticas lo que facilitó la interpretación y comprensión de los mismos.

Durante el desarrollo del estudio se cumplieron los principios éticos estipulados en la Declaración de Helsinki 2 para realizar investigaciones en seres humanos. Los datos fueron utilizados únicamente con fines investigativos y se tomaron medidas de confiabilidad de la información que incluyeron la no utilización de datos de identidad personal, la utilización de códigos alfanuméricos y la eliminación de la base de datos con toda la información después de realizado el informe final de la investigación. La incorporación de los pacientes al estudio fue totalmente voluntaria, sin que representara gasto algunos para ellos y con la posibilidad de retirarse de la investigación en el momento que lo consideraran necesario, cada paciente fue informado de los objetivos y métodos de la investigación antes de decidirse su incorporación al estudio. La incorporación de los pacientes se realizó únicamente después de firmar el consentimiento informado como muestra de si deseo de participar en el estudio.

\section{RESULTADOS Y DISCUSIÓN}

Tabla I. Distribución de pacientes según características epidemiológicas

\begin{tabular}{lc}
\hline $\begin{array}{c}\text { Características } \\
\text { epidemiológicas }\end{array}$ & $\begin{array}{c}\text { Muestra total de 78 } \\
\text { pacientes con ERC } \\
\text { Frecuencia (porciento) }\end{array}$ \\
\hline $\begin{array}{c}\text { Promedio de edad } \\
\text { Grupo de edades }\end{array}$ & $\begin{array}{c}54,68 \text { años *DE 18,32 } \\
\text { 20 a 30 años }\end{array}$ \\
31 a 40 años & $7(6,41)$ \\
41 a 50 años & $7(8,97)$ \\
51 a 60 años & $20(16,67)$ \\
61 años y más & $33(42,31)$ \\
& Sexo \\
Masculino & $31(39,74)$ \\
Femenino & $47(60,26)$
\end{tabular}

Autoidentificación étnica
Mestizo
$66(84,61)$

Indígena

$12(15,39)$

\section{Nivel de instrucción}

$\begin{array}{lc}\text { Analfabeto } & 7(8,97) \\ \text { Primaria incompleta } & 17(21,79) \\ \text { Primaria completa } & 30(38,46) \\ \text { Bachillerato } & 18(23,08) \\ \text { Superior } & 6(7,69)\end{array}$

Nivel económico personal

$\begin{array}{lc}\text { Ninguno } & 47(60,26) \\ \text { Bajo } & 25(32,05) \\ \text { Medio } & 6(7,69) \\ \text { Alto } & 0(0,00 \\ & \text { Procedencia } \\ \text { Rural } & 34(43,59) \\ \text { Urbano } & 44(56,41)\end{array}$

Presencia de comorbilidades

$\mathrm{Si}$

$77(98,72)$

No

Tipo de comorbilidades $n=77$ pacientes

Diabetes mellitus

$27(35,06)$

Hipertensión arterial

$71(92,21)$

Lupus eritematoso

$3(3,90)$

Uropatía obstructiva

$3(3,90)$

$* *$ EPOC

$1(1,30)$ 


\begin{tabular}{ll} 
Artritis reumatoide & $1(1,30)$ \\
Hipotiroidismo & $1(1,30)$ \\
$\begin{array}{l}\text { Hipertrofia } \\
\text { prostática }\end{array}$ & $2(2,60)$ \\
Otras & $4(5,20)$ \\
\hline
\end{tabular}

*DE: desviación estándar

**EPOC: Enfermedad pulmonar obstructiva crónica

La tabla I muestra las características epidemiológicas de los pacientes con diagnóstico de ERC atendidos en el HPGDR Se observa un promedio de edad de 54,68 años en el momento de realizar la investigación con predominio de pacientes de 61 años o más $(42,31 \%)$, seguido de los pacientes entre $51 \mathrm{y}$ 60 años de edad $(25,64 \%)$ y entre 41 y 50 años $(16,67 \%)$. En relación al sexo predominaron los pacientes del sexo femenino $(60,26 \%)$ en relación a los pacientes masculinos $(39,74 \%)$. El $56,41 \%$ de los pacientes refirió proceder de área urbana y el restante $43,59 \%$ procedía de área rural.

El $84,61 \%$ de los pacientes se autoidentificó como mestizos y el 15,39\% se autoidentificó como indígenas, siendo estas dos categorías las dos únicas que tuvieron representación en el estudio. También fue incluida dentro de las características epidemiológicas el nivel de instrucción y los ingresos económicos. El análisis del nivel de instrucción mostró un predominio de personas con primaria terminada $(38,46 \%)$, seguido de pacientes con bachillerato vencido $(23,08 \%)$ y primaria incompleta $(21,79 \%)$. Los resultados del análisis de los ingresos económicos personales mostraron que el $60,26 \%$ de los pacientes con ERC no presentaban, en elmomento de realizar la investigación, ingresos económicos fijos; el 32,05\% presentaba ingresos económicos bajos (menos de un salario básico mínimo equivalente a 400,00 dólares mensuales) y el restante $7,69 \%$ refirió ingresos económicos personales medios (entre 1 y 2 salarios mínimos mensuales) (tabla I).

Los últimos aspectos analizados dentro de la variable características epidemiológicas fueron la presencia y tipo de comorbilidades en los pacientes con ERC. En este sentido se determinó que el 98,72\% de los pacientes presentaba al menos una comorbilidad asociada, y dentro de estas la hipertensión arterial (92,21\%) y la diabetes mellitus $(35,06 \%)$ fueron las comorbilidades que con mayor frecuencia fueron referidas por los pacientes (tabla I).

Tabla II. Distribución de pacientes según características clínicas de la enfermedad

\begin{tabular}{lcc}
\hline $\begin{array}{l}\text { Características } \\
\text { clínicas }\end{array}$ & $\begin{array}{c}\text { Muestra total de 78 pacientes } \\
\text { con ERC }\end{array}$ \\
& Frecuencia $\quad$ Por ciento \\
\hline
\end{tabular}

\begin{tabular}{lcc}
\hline & Estado nutricional \\
Bajo peso & 17 & 21,79 \\
Normo peso & 33 & 42,31 \\
Sobrepeso & 19 & 24,36 \\
Obesidad & 9 & 11,54 \\
& $\begin{array}{c}\text { Tiempo de } \\
\text { evolución }\end{array}$ \\
Menor de 1 año & 7 & 8,97 \\
De 1 a 5 años & 38 & 48,72 \\
De 6 a 10 años & 19 & 24,36 \\
Más de 10 años & 14 & 17,95
\end{tabular}

\section{Tipo de manifestaciones clínicas}

$\begin{array}{lll}\begin{array}{l}\text { Edema } \\ \text { miembros } \\ \text { inferiores }\end{array} & 36 & 46,15 \\ \begin{array}{l}\text { Hipertensión } \\ \text { arterial }\end{array} & 71 & 92,21 \\ \text { Anemia } & 23 & 29,49 \\ \text { Anorexia } & 19 & 24,36 \\ \begin{array}{l}\text { Vómitos } \\ \text { nauseas }\end{array} & 15 & 19,23 \\ \text { Fracturas óseas } & 4 & 5,13\end{array}$




\begin{tabular}{|c|c|c|}
\hline Polineuropatía & 9 & 11,54 \\
\hline \multicolumn{3}{|c|}{ Uso de terapia sustitutiva } \\
\hline $\mathrm{Si}$ & 78 & 100,0 \\
\hline No & 0 & 0,00 \\
\hline \multicolumn{3}{|c|}{ Tipo de terapia sustitutiva } \\
\hline $\begin{array}{l}\text { Diálisis } \\
\text { peritoneal }\end{array}$ & 4 & 5,13 \\
\hline Hemodiálisis & 74 & 94,87 \\
\hline
\end{tabular}

En la tabla II se muestra la distribución de los pacientes con ERC según las características clínicas de la enfermedad. Se identificó que $48,72 \%$ de los casos llevaba entre 1 y 5 años de diagnosticado la ERC, el 24,36\% entre 6 y 10 años y el 17,95\% presentaba más de 10 años de evolución de la enfermedad; solo el $8,97 \%$ presentaba menos de un año de evolución de la enfermedad. A pesar de que el $42,31 \%$ de los pacientes presente un estado nutricional normal (normo peso), se identificó un $24,36 \%$ de pacientes con sobrepeso, un $21,79 \%$ de pacientes presentó bajo peso y el restante $11,54 \%$ obesidad.

La hipertensión arterial (92,21\%) fue la manifestación clínica que con mayor frecuencia fue referida por los pacientes. También fue referido en el $46,15 \%$ de los casos la presencia de edemas en miembros inferiores; el 29,49\% presentaba anemia, el $24,36 \%$ anorexia, el 19,23\% refirió vómitos y diarreas, el 11,54\% refirió cuadro clínico compatible con diagnóstico de polineuropatía $\mathrm{y}$ en el $5,13 \%$ se habían presentado fracturas óseas. La totalidad de los pacientes investigados usaban terapia sustitutiva; de ellos 4 pacientes $(5,13 \%)$ se encuentran recibiendo diálisis peritoneal, mientras que el $94,87 \%$ se encuentra incluido dentro del programa de hemodiálisis (tabla 2). La frecuencia de realización de hemodiálisis en la totalidad de los pacientes era de tres veces por semana

\section{Discusión}

Conocer las características clinicoepidemiológico de la ERC permite a los profesionales de la salud tener elementos que facilitan la orientación diagnóstica y terapéutica ante la presencia de manifestaciones clínicas y complicaciones relacionadas con esta enfermedad. (Terazón Miclín, Vinent Terazón, \& Pouyou Semanat, 2017). Es por eso que esta investigación, primera de su tipo en el contexto de la investigación y referente a nivel provincial y nacional, abre el camino a otros estudios más profundos sobre la ERC que incluyan otros aspectos de la enfermedad como esquemas terapéuticos, medidas intervencionistas $\mathrm{y}$ percepción de calidad de vida entre otros.

El análisis de las características epidemiológicas de los pacientes investigado mostró algunos resultados validos de destacar. El primero de ellos se relaciona directamente con la edad de diagnóstico de la enfermedad. A pesar de existir un predominio de personas con 60 años o más, lo cual es un dato reportado en otras investigaciones (Castellanos Castillo, et al., 2018), llama la atención el elevado porcentaje de personas con diagnóstico de ERC y edad inferior a los 40 años. Esta situación pudiera estar relacionada con el aumento de factores de riesgo de aparición de la ERC y la baja percepción de riesgo de la población hacia esta patología. Sin embargo, se necesitarían otras investigaciones para poder confirmar esta hipótesis. 
Otro resultado que difiere de los reportes internacionales es el predominio de mujeres con diagnóstico confirmado de ERC. Autores como Pinillos-Patiño y colaboradores (2019) describen un predominio de incidencia de la enfermedad en pacientes masculinos. Una posible explicación a este resultado puede estar dado por una deficiente identificación de los factores de riesgo de aparición de ERC; en este sentido sorprende las dos primeras causas de afección renal en el sexo masculino, la artropatía gotosa y el síndrome metabólico, no fueron identificadas dentro de las comorbilidades de los pacientes masculinos. Este hecho muestra que aún queda mucho camino por recorres en pos de lograr una atención primaria de salud, donde los principales ejes de acción se orientan hacia la promoción de salud y la prevención de enfermedades.

También se analizó el nivel de instrucción de las personas investigadas y el nivel económico personal. Si bien es cierto que no forman parte de los factores de riesgo reportados para la ERC, ambos pueden influir en el control de la enfermedad, sobre todo en relación con el autocuidado y la adherencia farmacológica. En el caso del nivel de instrucción se reporta que mientras es mayor el nivel educacional de la población también es mayor la adherencia farmacológica y los cuidados característicos de la enfermedad ya que aumenta, entre otros elementos, la percepción de riesgo de los pacientes, adoptando conductas más responsables en cuanto al cuidado de salud se refiere (Costa-Requena, Moreso, Cantarell, \& Serón, 2017).

En relación con el nivel económico personal de los pacientes es necesario destacar que la ERC es una afección que demanda elevados gastos relacionados con el proceso salud enfermedad. La adquisición de fármacos, la asistencia a controles regulares, realización frecuente de exámenes complementarios y los requerimientos nutricionales son algunas de las situaciones que demandan recursos económicos. Autores como Terazón Miclín, Vinent Terazón y Pouyou Semanat, (2017) reportan que la ERC es una enfermedad que demanda de los pacientes y familiares disponer de recursos económicos para solventar las necesidades de atención médica. Por lo tanto, la solvencia económica es un elemento que facilita en gran medida la compra de medicamentos, asistencia a consultas y adecuado seguimiento, factores estos que pueden marcar 1 diferencia en cuento a control de la actividad clínica y prevención de complicaciones se refiere.

El elevado por ciento de pacientes con comorbilidades previo al diagnóstico de la ERC coincide con lo señalado por Aleman Vega y colaboradores (2017). Este resultado evidencia que sin dudas es la ERC una enfermedad que se produce secundariamente a determinados procesos que tienen como elemento común el daño renal con disminución considerable del funcionamiento de este, generando entonces las manifestaciones clínicas de la enfermedad que permiten sospechar inicialmente $\mathrm{y}$ confirmar posteriormente el diagnóstico de la enfermedad (Garrido Blanco, Arroyo Priego, Arana Ruiz, López Zamorano, Tierno Tendero, \& Crespo Montero, 2018).

Como ha sido descrito históricamente la 
hipertensión arterial y la diabetes mellitus, son de forma general las enfermedades que con mayor frecuencia generan daño renal predictor de ERC; sin embargo, destaca la presencia de otras afecciones menos comúnmente presentes en la población pero que su presencia implica un riesgo elevado de afectación renal; estas son el lupus eritematoso sistémico, las uropatías obstructivas y la artritis reumatoide (Garrido Blanco, et al, 2018).

El análisis de los elementos clínicos de la ERC muestra un tiempo de evolución de la enfermedad que coincide con el de otros reportes (Díaz Armas, et al, 2018). Es importante destacar que mucho de los pacientes con ERC tienen como destino final el trasplante renal, por lo que el tiempo de evolución de le enfermedad es variable y cambiante. Sin embargo, en no pocas ocasiones los pacientes permanecen un periodo prolongado de tiempo en las listas de trasplantes. Adicionalmente es necesario mencionar que la evolución crónica de la enfermedad depende de muchos factores, por lo que es imposible definir qué tiempo transcurrirá desde el diagnostico hasta la incorporación a la terapia sustitutiva y menos aún hasta la fecha de realizar el trasplante renal (Gutiérrez Sánchez, Leiva-Santos, Macías López, \& Cuesta Vargas, 2017).

Un elemento negativo encontrado es el elevado por ciento de pacientes con alteraciones nutricionales, principalmente por exceso, lo cual es un elemento característico del perfil nutricional de la población ecuatoriana (Ramos-Padilla, Carpio-Arias, DelgadoLópez, \& Villavicencio-Barriga, 2015).
Los trastornos nutricionales por exceso (sobrepeso $\mathrm{u}$ obesidad) condicionan un estado de hiperlipemia e hiperglucemia que generan mayor daño renal; por lo que resulta fundamental la orientación nutricional en los pacientes con ERC para minimizar, mediante un estado nutricional adecuado, el daño de la función renal.

Los síntomas y signos que con mayor frecuencia fueron referidos por los pacientes o identificados en la historia clínica fueron la hipertensión arterial, los edemas en miembros inferiores y la presencia de anemia; estos son descritos como la triada clásica de la enfermedad (Gutiérrez Sánchez, LeivaSantos, Macías López, \& Cuesta Vargas, 2017). Un resultado que destaca es el reporte de fracturas por parte de los pacientes; aunque no fue posible comparar el dato con otras investigaciones si se considera necesario recordar que la ERC es una enfermedad que afecta el metabolismo normal del calcio; de esta forma disminuye la densidad mineral ósea y el hueso se hace más débil perdiendo resistencia; estos elementos condicionan el aumento de fracturas patológicas como consecuencia de una osteoporosis secundaria (Gutiérrez Sánchez, Leiva-Santos, Macías López, \& Cuesta Vargas, 2017).

\section{IV.- CONCLUSIONES}

De forma general el patrón clínico epidemiológico de los pacientes con ERC atendidos en el HPGDR durante el primer semestre del año 2021 coincide con lo reportado en la literatura. Existió un predominio de personas mayores de 50 años, 
con comorbilidades asociadas y donde la hipertensión arterial, los edemas en miembros inferiores y la anemia fueron los elementos clínicos que con mayor frecuencia se identificaron. Sin embargo, existen elementos como la presencia de la enfermedad en pacientes menores de 40 años y el elevado por ciento de trastornos nutricionales por exceso que constituyen señales de alarma para los directivos de salud y para los profesionales en torno a la promoción de salud y prevención de enfermedades.

\section{V.- REFERENCIAS BIBLIOGRÁFICAS}

Alemán-Vega, G., Gómez Cabañas, I., Reques Sastre, L., Rosado Martín, J., Polentinos-Castro, E., \& Rodríguez Barrientos, R. (2017). Prevalence and risk of progression of chronic kidney disease in diabetics and hypertensive patients followed in primary care in Madrid. Nefrología (Madrid), 37(3), 343-345. Recuperado de https://dx.doi. org/10.1016/j.nefro.2016.10.019

Castellanos Castillo, Y., Fong Estrada, J.A., Vázquez Trigo, J.M., \& Fong, J. (2018). Marcadores de daño renal en pacientes con factores de riesgo de enfermedad renal crónica. MEDISAN, 22(2), 142-148. Recuperado de http://scielo.sld.cu/scielo. php? script $=$ sci_arttext\&pid=S102930192018000200004\&lng=es\&tlng=es

Costa-Requena, G., Moreso, F., Cantarell, M.C.,\&Serón,D.(2017).Alfabetización en salud y enfermedad renal crónica.
Nefrología (Madrid), 37(2), $115-$ 117. Recuperado de https://dx.doi. org/10.1016/j.nefro.2016.10.001

Díaz Armas, M.T., Gómez Leyva, B., Robalino Valdivieso, M.P., \& Lucero Proaño, S.A. (2018). Comportamiento epidemiológico en pacientes con enfermedad renal crónica terminal en Ecuador. Correo Científico Médico, 22(2), 312-324. Recuperado de http://scielo.sld.cu/scielo. php?script $=$ sci_arttext\&pid $=\mathrm{S} 1560$ 43812018000200011\&lng=es\&tlng=es

Gansevoort, R., Correa-Rotter, R., Hemmelgarn, B., Jafar, T., Heerspink, H., Mann, J., Matsushita, K., \& Wen, C. (2013): Chronic kidney disease and cardiovascular risk: epidemiology, mechanisms, and prevention. Lancet; 382: 339-352

Garrido Blanco, R., Arroyo Priego, E., Arana Ruiz, A.I., López Zamorano, M.D., Tierno Tendero, C., \& Crespo Montero, R. (2018). Quality of life and advanced chronic kidney disease. Influence of renal clearance. Enfermería Nefrológica, 21(4), 359367. https://dx.doi.org/10.4321/s225428842018000400005

Gutiérrez Sánchez, D., Leiva-Santos, J.P., Macías López, M.J., \& Cuesta Vargas, A.I. (2017). Perfil sintomático de los pacientes con Enfermedad Renal Crónica Estadio 4 y 5 . Enfermería Nefrológica, 20(3), 259-266. https://dx.doi.org/10.4321/s225428842017000300010 
Gutiérrez Rufín, M., \& Polanco López, C. (2018). Enfermedad renal crónica en el adulto mayor. Revista Finlay, 8(1), 1-8. Recuperado en 14 de abril de 2021, de http://scielo.sld.cu/scielo. php? script $=$ sci_arttext\&pid $=$ S2221 $24342018000100001 \& \operatorname{lng}=$ es\&tlng=es

Mora Marcial, G.R., Verdecia Tamallo, K., Rodríguez Vergara, T.M., del Pino, B.N., \& Guerra Cabrera, C. (2017). Adherencia terapéutica en pacientes con algunas enfermedades crónicas no transmisibles. Revista Cubana de Medicina General Integral, 33(3), 270-280. Recuperado de http://scielo.sld.cu/scielo. php? script $=$ sci_arttext\&pid $=$ S0864$21252017000300001 \& \operatorname{lng}=$ es\&tlng=pt

Otzen, T., Fuentes, N., Wetzel, G., Henríquez, C., Antúnez, Z., \& Melnik, T. (2020). Suicidality and perceived social support in university students with chronic non-communicable diseases. Terapia psicológica, 38(1), 119-129. https://dx.doi.org/10.4067/S071848082020000100119

Pinillos-Patiño, Y., Herazo-Beltrán, Y., Gil Cataño, J., \& Ramos de Ávila, J. (2019). Actividad física y calidad de vida en personas con enfermedad renal crónica. Revista médica de Chile, 147(2), 153-160. Recuperado de https://dx.doi.org/10.4067/s003498872019000200153

Poll Pineda, J.A., Rueda Macías, N.M., Poll Rueda, A., Mancebo Villalón, A., \& Arias Moncada, L. (2017). Factores de riesgo asociados a la enfermedad renal crónica en adultos mayores. MEDISAN, 21(9), 2010-2017. Recuperado de http://scielo.sld.cu/scielo. php? script $=$ sci_arttext\&pid $=$ S 102930192017000900006\&lng=es\&tlng=es

Ramos-Padilla, P., Carpio-Arias, T., DelgadoLópez, V., \& Villavicencio-Barriga, V. (2015). Sobrepeso y obesidad en escolares y adolescentes del área urbana de la ciudad de Riobamba, Ecuador. Revista Española de Nutrición Humana y Dietética, 19(1), 21-27. https://dx.doi. org/10.14306/renhyd.19.1.123

SerraValdés,M.,SerraRuíz,M.,\&VieraGarcía, M. (2018). Las enfermedades crónicas no transmisibles: magnitud actual y tendencias futuras. Revista Finlay, 8(2), 140-148. Recuperado en 14 de abril de 2021, de http://scielo.sld.cu/scielo. php? script $=$ sci_arttext\&pid $=$ S2221 24342018000200008\&lng=es\&tlng=es

Terazón Miclín, O., Vinent Terazón, M.A., \& Pouyou Semanat, J. (2017). Determinación del grado de enfermedad renal crónica en pacientes hipertensos. MEDISAN, 21(1), 1926. Recuperado en 14 de abril de 2021, de http://scielo.sld.cu/scielo. php?script $=$ sci_arttext\&pid $=$ S 1029 $30192017000100003 \& \operatorname{lng}=\mathrm{es} \& \operatorname{lng}=\mathrm{es}$

Torres Rondón, G., Bandera Ramos, Y., Ge Martínez, P.Y., \& Amaro Guerra, I. (2017). Factores de riesgo de enfermedad renal crónica en pacientes del municipio de II Frente. MEDISAN, 21(3), 265-272. Recuperado 
de

http://scielo.sld.cu/scielo.

php? script $=$ sci_arttext\&pid $=$ S1029-

30192017000300004\&lng=es\&tlng=es 